\title{
Fahreignungsbeurteilung älterer Autolenker - Bedeutung der Fahrberatung
}

Karl Seiler

Ausbildung «Fahrberater SFV»

Korrespondenz:

Dr. med. Karl Seiler

Innere Medizin FMH

Obere Hofbergstrasse 5

CH-9500 Wil

kmseiler@bluewin.ch

\section{Einleitung}

Ein Erwachsener darf ein Auto lenken, wenn er allgemein vom körperlichen, geistigen und psychischen Zustand aus dafür geeignet ist (Fahreignung) und wenn er vor der Fahrt sich in einem guten gesundheitlichen Zustand (Fahrtüchtigkeit) befindet und eine genügend gute fahrtechnische Fertigkeit (Fahrkompetenz) hat. Mittels der amtlichen Fahrprüfung, die man meist in jungen Jahren absolviert, wird in erster Linie die in der Fahrschule erworbene technische Fertigkeit zum sicheren Lenken eines Fahrzeugs im Strassenverkehr kontrolliert. Im Alter ab 70 möchte man mit einem ärztlichen Attest die Fahreignung bestätigt haben. Es soll festgestellt werden, ob die medizinischen Mindestanforderungen gemäss «Verkehrszulassungsverordnung» (VZV) erfüllt sind. Leider ist die heutige Praxissituation unbefriedigend. Besonders ist der Katalog für die Führerausweisanforderung von älteren Fahrern unbefriedigend. Die kantonal verschiedenen Formulare für die ärztliche Fahreignungsbeurteilung ab 70 widerspiegeln die amtlichen Unsicherheiten. Weil aus Sicht der praxisnahen Fahreignungskontrolle die Prüfung der fahrtechnischen Befähigung nicht einfach vernachlässigt werden darf, bilden Hausärzte in Zusammenarbeit mit dem Schweizerischen Fahrlehrerverband Fahrberater für Senioren aus.

\section{Bedeutung der Fahrberatung}

Die Beurteilung eines autolenkenden Seniors ob dieser nun wegen vorübergehender gesundheitlicher Störung oder mit dem Wunsch zur Durchführung der amtlich verlangten ärztlichen Fahreignungskontrolle in die Sprechstunde kommt - verlangt vom Arzt besondere Sorgfalt, umfassendes Wissen und Kenntnisse des Patientenumfeldes. Alte Menschen wissen aus Erfahrung, wie schnell sich die Leistungsfähigkeit ändern kann und die Fahrtüchtigkeit ungenügend wird. Wenn immer der Gesundheitszustand eines autolenkenden Patienten sinkt, wird der Hausarzt auch die zukünftige Fahreignung sorgfältig überprüfen und nachkontrollieren.

Weil das Resultat einer ärztlichen Untersuchung nicht in jedem Fall mit dem Resultat am Steuer übereinstimmen muss, haben Hausärzte und interessierte Fahrlehrer die Initiative ergrif-

\section{Examen d'aptitude à la conduite des automobilistes âgés - importance des conseils en circulation routière}

Un adulte ne peut conduire de voiture que si son état physique, mental et psychique le permet, s'il est en bonne santé (aptitude à la conduite) et si ses compétences techniques en la matière sont suffisantes (aptitudes du conducteur). L'examen de conduite - que l'on passe généralement durant ses jeunes années - a principalement pour but de vérifier les compétences techniques acquises durant les cours d'auto-école pour circuler en toute sécurité. A partir de 70 ans, I'aptitude à la conduite devrait être attestée par un certificat médical, l'objectif visé étant de vérifier si les exigences médicales minimales selon I'Ordonnance réglant l'admission à la circulation routière $(\mathrm{OAC})$ sont bien remplies. Pour que ce contrôle ne conduise pas à négliger les aptitudes du conducteur, les médecins de premier recours et I'Association suisse des moniteurs de conduite forment des moniteurs spécialisés pour le troisième âge.

fen, älteren Fahrern eine freiwillige Fahrberatung anzubieten. Die Ausbildung zum Fahrberater ist anspruchsvoll, und sie wird unter der Leitung des Schweizerischen Fahrlehrerverbandes, von Hausärzten und themenbezogenen Spezialisten durchgeführt. Ärzte schicken nur Probanden, die die medizinischen Mindestanforderungen gemäss VZV erfüllen, zur Fahrberatung.

\section{Die Ausbildung zum Fahrberater}

Die Ausbildung dauert vier Monate. An vier Kurstagen lernen die Berater zehn für das Fahren wichtige gesundheitliche Problemkreise der älteren Lenker kennen. Sie sollen die Krankheiten auf einem Niveau eines gutinformierten Patienten kennen. Sie studieren, wie sich Altersschwä- 
che und Krankheit auf die Fahrweise auswirken. Zwei Kurstage sind für praktische Fahrberatungen älterer Probanden/-innen und für Vorträge von Spezialisten reserviert. Am Schlusstag sind Prüfungen, themenübergreifende Vorträge und Diskussionen. Die Weiterbildung der Fahrberater erfolgt über die Vereinigung der «Fahrberater SFV».

\section{Wie läuft eine Fahrberatung ab?}

$\mathrm{Zu}$ Beginn der freiwilligen, gut einstündigen Probefahrt erkundigt sich der Fahrberater nach dem Wohlbefinden des Seniors und nach möglichen Fahrproblemen. Dann folgen die Fahrprobe und die Besprechung wichtiger Fragen. Die Fahrt findet mit dem Fahrzeug des Seniors statt. Zum Schluss beantworten Fahrer und Berater gemeinsam die Fragen auf einem einheitlichen Beratungsformular. Der Hausarzt erhält auf Wunsch des Seniors eine Berichtkopie.

Die Fahrt ist in zehn Abschnitte aufgeteilt. Jeder Abschnitt entspricht einem gesundheitlichen Themenkreis, der die Fahrweise negativ beeinflussen könnte (Abbildung).

- Auf dem ersten, einfachen Streckenabschnitt zeigt der Fahrer seine Fahrroutine, er kennt die Verkehrsregeln, und er vermittelt dem mitfahrenden Fahrberater ein sicheres Gefühl.

- Als zweites kommt der Fahrer in eine Gegend mit schwierigen Sichtverhältnissen. Strassenschilder sind bei gutem Licht leichter lesbar als bei Dämmerung. Eine Gefahr soll schnell wahrgenommen werden.

- Komplizierter wird der dritte Streckenabschnitt. Auf Anordnung des Fahrberaters wird eine Mehrfachaufgabe, z.B. Durchfahren einer Kreuzung mit Kreisverkehr und anschliessend Überquerung einer Kreuzung mit verschiedenen Markierungen und Signalen, verlangt. Die Merkfähigkeit und Bewältigung komplexer Situationen werden damit gezeigt.

- Im vierten Abschnitt geht es auf einer Nebenstrasse über Land oder durch ein ruhigeres Gebiet. Dynamische Beweglichkeit, Spurhaltung, feines Reagieren und auf Befehl eine Notbremsung werden verlangt.

- Als fünfte Aufgabe soll der Senior auf einem Parkplatz das Rückwärtsfahren und Parkieren demonstrieren. Gute Tips vom Berater sind hier willkommen. Anschliessend bietet sich Gelegenheit für eine Fahrpause.

- Als Punkt sechs und sieben folgen Gespräche über zwei wichtige gesundheitliche Themen. Die häufigsten gesundheitlichen Störungen im Alter, welche die Fahrtüchtigkeit manch- mal unmerklich einschränken, sind die Herzund Kreislaufkrankheiten. Fahren nur bei Wohlbefinden und Achten auf schädliche Risikofaktoren wie Rauchen, fettes Essen usw. ist sehr wichtig. Ein bedeutendes Problem am Steuer entsteht bei plötzlich auftretendem Unwohlsein. Bei Senioren mit Alterszucker wird der Fahrberater die entsprechenden Vorsichtsmassnahmen diskutieren.

- Nach der Weiterfahrt wird auf dem achten Streckenabschnitt eine eigene Notfallsituation angenommen, z. B. eine Bewusstseinstrübung mit Sehstörung wegen Zirkulationsstörung. Das richtige Verhalten in der Extremsituation wird geübt.

- Auf dem Rückweg werden als neunter und zehnter Punkt mit dem Fahrer die Probleme des Alkohols und gefährlicher Medikamente angesprochen.

\section{Resultat der Fahrberatung}

Anlässlich der Probefahrt lernt der Senior seine eigenen Stärken und Schwächen kennen. Zum Beispiel wird ihm seine Sehschwäche unter schlechten Lichtverhältnissen eindrücklich demonstriert. Manchmal wird der Fahrer bei einer Fahrt über die Autobahn infolge des lebhaften Verkehrs etwas verängstigt, oder die Konzentration lässt nach Fahrt im dichten Verkehr schnell nach. Für den älteren Fahrer werden Vorsichtsmassnahmen wie «Fahren nur tags» - «Fahren nur bei guten Lichtverhältnissen»- «Autobahn und Stossverkehr meiden» sehr wichtig. Manche Tips, zum Beispiel betreffend Sitzeinstellung, Position der Aussen- und Innenspiegel, und Erholungsmöglichkeiten helfen, die Fahrtüchtigkeit zu verbessern.

Bei jedem Fahrer aber kommt einmal der Zeitpunkt, dass infolge Schwäche und Krankheit die Fahrtüchtigkeit zu stark eingeschränkt wird. Es ist dann besser, den Führerausweis beim Strassenverkehrsamt abzugeben. Die eigene Einsicht, Ratschläge von Angehörigen und guten Bekannten, Gespräche mit dem Hausarzt oder vielleicht eine Fahrberatung helfen, den Entscheid des Führerausweisverzichtes rechtzeitig $\mathrm{zu}$ fällen. «Ein entzogener Führerausweis bedrückt den Senior fast lebenslänglich - ein von sich aus zurückgegebener Führerausweis erfüllt ihn zu Recht mit Stolz», dies ist die Erfahrung Hansueli Bleikers, des Pioniers der Fahrberater.

Die Möglichkeit der verkehrsmedizinischen Spezialuntersuchung bei schwierigen verkehrsmedizinischen Problemen oder bei uneinsichtigen Patienten stellt eine wertvolle Ergänzung zur Fahreignungsbeurteilung im Praxisalltag und auch anlässlich der amtlichen vertrauens- 


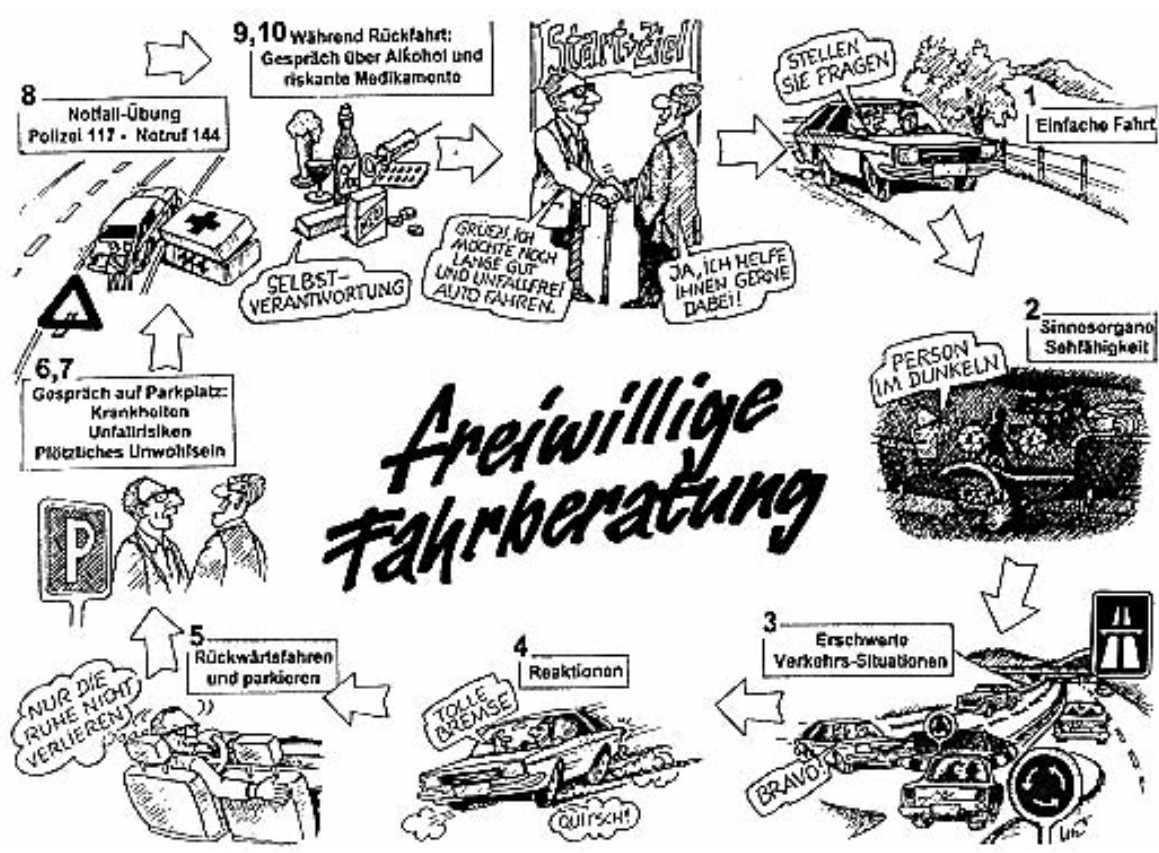

ärztlichen Kontrolluntersuchung ab einem Alter von 70 Jahren dar.

\section{Kann der Fahrberater einem Senior den Führerausweis entziehen?}

Nein, der Fahrberater hält nur den momentanen Befund der Fahrtüchtigkeit fest, das heisst, er hält das Resultat der Fahrprobe mit den spezifisch festgestellten Schwächen fest, er notiert die persönlichen Tips und die für die Sicherheit relevanten Verbesserungsvorschläge. Wichtig ist die Festlegung individueller Vorsichtsmassnahmen.

Wenn bei der Fahrprobe zu grosse Defizite zum Vorschein gekommen sind, wird der betagte Autofahrer vom Berater sanft, aber überzeugend auf die Vorteile des freiwilligen Verzichtes aufmerksam gemacht. Der Fahrberater ist immer nur Berater, die Entscheidung über die Fahreignung, sofern sie nicht frühzeitig vom Fahrer selbst gefällt wird, treffen der Arzt bzw. die amtlichen Kontrollorgane.

\section{Was kostet die Beratung, und wie finde ich einen guten Fahrberater?}

Die Kosten für eine Fahrberatung entsprechen ungefähr jenen von zwei Fahrlektionen. Fahrberatungen sind freiwillig. Sie werden von älteren Fahrern spontan gewünscht, manchmal vom Arzt empfohlen, selten als Vorabklärung oder Vorbereitung vor einer amtlichen Kontrollfahrt gemacht.
Bisher wurden rund 120 Fahrberater/innen ausgebildet. Schwerpunktmässig kommen sie aus der deutschsprachigen, besonders aus der Ostschweiz. Bisher nur Vereinzelte stammen aus der französisch- und italienischsprachigen Schweiz. Die vom Schweizerischen Fahrlehrerverband anerkannten «Fahrberater SFV» findet man über Internet: www.fahrberater-sfv.ch.

Anmerkung: Bei der obligatorischen vertrauensärztlichen Fahreignungskontrolle ab 70 soll wegen amtlicher juristischer Bedenken eine allfällig vorher durchgeführte freiwillige Fahrberatung nicht gewertet werden. Der Hausarzt als Gutachter des Strassenverkehrsamtes habe sich ausschliesslich zur medizinischen Fahreignung zu äussern (asa, Strassenverkehrsamt).

\section{Literatur}

- Seeger R. Probleme bei der Beurteilung der Fahreignung in der hausärztlichen Praxis. Ther Umschau. 1997;54(5):242-54.

- Seiler K. Beurteilung der Fahrtauglichkeit älterer und kranker Personen. PrimaryCare. 2004;4(14): 290-2.

- Haag M, Dittmann V. Handbuch der verkehrsmedizinischen Begutachtung. Bern: Hans Huber; 2005.

- Kissling B. Fahrfähigkeitsbeurteilung von Senioren - Sorgfalt und Verantwortung. PrimaryCare. 2007; $7(20 / 21): 322$. 